\title{
ON SLANT IMMERSIONS INTO KÄHLER MANIFOLDS
}

\author{
By Sadahiro Maeda, Yoshihiro Ohnita and Seitchi Udagawa
}

\section{Introduction.}

Let $\varphi: M \rightarrow N$ be an isometric immersion of a Riemannian manifold into an almost Hermitian manifold with almost complex structure $\tilde{J}$. Then, $\varphi$ is called slant if the angle between $\tilde{J} \varphi_{*}(X)$ and $\varphi_{*}\left(T_{p} M\right)$ is constant for any $X \in T_{p} M$ and any $p \in M$. The typical examples of slant immersions are Kähler immersions and totally real immersions, where the slant angles are 0 and $\pi / 2$, respectively. A slant immersion is called proper if it is neither a Kähler immersion nor a totally real immersion. In the case where $M$ is a Riemann surface and $N$ is a Kähler manifold, the slant angle was introduced as the Kähler angle and studied by S. S. Chern and J. G. Wolfson [CW]. Examples of slant immersions of a Riemann sphere $S^{2}$ into a complex projective space $C P^{n}$ were given as the Veronese sequence of harmonic maps from $S^{2}$, which are classified in [BO] and [BJRW] in the case where $S^{2}$ has constant curvature (see also [O1]). The present concept of slant immersion was first introduced and studied by B.Y. Chen [C]. The examples of proper slant immersions into $C^{4}$ are given in [C-T]. Recently, Tazawa [T] has given examples of slant immersions into $\boldsymbol{C}^{n}$ with any given slant angle. However, there are a few results on slant submanifolds in $C P^{n}$. In this case, any general method to check whether given an immersion is slant or not is not known.

The main purpose of this paper is to study slant submanifolds in $C P^{n}$. In Section 1, we give some sufficient conditions for an isometric immersion $\varphi$ of a compact Kähler manifold $M$ into a Kähler manifold $N$ to be slant (Theorem 1.2, Proposition 1.3). In Theorem 2.1 of Section 2, we show that the condition of Theorem 1.2 is satisfied for a G-equivariant isometric immersion of a Kähler C-space $M$ with $b_{2}(M)=1$ into $C P^{n}$. In this case, the slant angle is explicitly given by $\cos ^{-1}(4 \pi|\operatorname{deg}(\varphi)| / \tilde{c} \operatorname{vol}(S))$, where $S$ is a rational curve of $M$ which represents a positive generator of $\mathrm{H}_{2}(M ; \boldsymbol{Z})$ and $\tilde{c}$ is a (constant) holomorphic sectional curvature of $C P^{n}$. Consequently, it turns out that $\mathrm{SU}(m+1)$-equivariant isometric immersions of $C P^{m}$ into $C P^{N}$ constructed and treated by the first and second author $([\mathrm{M}],[\mathrm{O} 2])$ are slant, and that pluriharmonic maps constructed in [OU] give many examples of proper slant immersions into $\boldsymbol{C} P^{n}$.

Received July 20, 1992. 
In Section 3, we give an extension of a theorem obtained in [MU]. An isometric immersion $\varphi: M \rightarrow N$ is called circular geodesic if $\varphi$ sends any geodesic in $M$ into a circle in $N$. We here note that "circular geodesic" is equivalent to "helical geodesic of order 2" regardless of the ambient manifold $N$ (for details, see [M]). K. Sakamoto [S] classified such immersions in case $N$ is a real space form. However, in case $N$ is a (non-flat) complex space form, the situation is quite different because of the presence of the complex structure of the target manifold. All the known examples of circular geodesic submanifolds in non-flat complex space forms are submanifolds with parallel second fundamental form, which are known to be Kähler or totally real. The first author and N. Sato [MS] proved that there are no other examples of circular geodesic submanifolds in the class of CR-submanifolds. A CR-submanifold is defined by the condition that its tangent space consists of the holomorphic distribution and the totally real distribution and the dimension of each distribution is constant over the submanifold. Hence, a CR-submanifold can not be a proper slant submanifold. Therefore, it is reasonable to look for circular geodesic submanifolds in the class of slant submanifolds. We prove that a circular geodesic slant submanifold with constant scalar curvature in a non-flat complex space form is a parallel submanifold, hence the immersion must be Kähler or totally real (Theorem 3.3).

The first author is partially supported by Ishida Foundation.

\section{§ 0. Preliminaries.}

Let $\varphi: M \rightarrow N$ be an isometric immersion of a Riemannian manifold into a Riemannian manifold. Let $\varphi_{*}: T M \rightarrow T N$ be the differential of $\varphi$, where $T M$ and $T N$ are the tangent bundles of $M$ and $N$, respectively. We often identify $\varphi_{*}(X)$ with $X$ itself, where $X \in T_{p} M$. Suppose that $N$ is an almost Hermitian manifold with almost complex structure $\tilde{J}$. Then, the slant angle $\theta_{X}(p)$ between $\varphi_{*}(X)$ and $\varphi_{*}\left(T_{p} M\right)$ is given by

$$
\cos \theta_{X}(p)=\frac{1}{\left|\pi \circ \tilde{J} \varphi_{*}(X)\right|}\left\langle\pi \circ \tilde{J} \varphi_{*}(X), \tilde{J} \varphi_{*}(X)\right\rangle \quad \text { with }|X|=1,
$$

where $\pi: T_{\varphi(p)} N \rightarrow \varphi_{*}\left(T_{p} M\right)$ is an orthogonal projection. Take an othonormal basis $\left\{\boldsymbol{e}_{i}\right\}$ of $T_{p} M$. Then we have $\pi \circ \tilde{J} \varphi_{*}(X)=\sum_{\imath=1}^{n}\left\langle\pi \circ \tilde{J} \varphi_{*}(X), \varphi_{*}\left(\boldsymbol{e}_{\imath}\right)\right\rangle \varphi_{*}\left(\boldsymbol{e}_{\imath}\right)$ and $\left|\pi \circ \tilde{J} \varphi_{*}(X)\right|=\cos \theta_{X}(p)$, where $n=\operatorname{dim} M$, so that $(0.1)$ becomes

$$
\cos ^{2} \theta_{X}(p)=\sum_{\imath=1}^{n}\left\langle\hat{J} \varphi_{*}(X), \varphi_{*}\left(\boldsymbol{e}_{\imath}\right)\right\rangle^{2} \quad \text { for } X \in T_{p} M \text { with }|X|=1 .
$$

Next, we return to the general situation that $N$ is a Riemannian manifold. Let $\tilde{\nabla}$ and $\nabla$ be the Riemannian connection and the induced connection of $N$ and $M$, respectively. The second fundamental form $\sigma$ of the immersion is defined by $\sigma(X, Y)=\tilde{\nabla}_{X} Y-\nabla_{X} Y$, where $X, Y \in T M$. The mean curvature vector is $(1 / n) \operatorname{tr}_{g} \sigma$, where $g$ is the Riemannian metric of $M$, and the mean curvature 
$H$ is the length of the mean curvature vector. If $H$ is identically zero, then the immersion $\varphi$ is called minimal. For a vector field $\xi$ normal to $M$, set $\tilde{\nabla}_{X} \xi$ $=-A_{\xi} X+D_{X} \xi$, where $-A_{\xi} X$ and $D_{X} \xi$ are the tangential and the normal components of $\tilde{\nabla}_{X} \xi$, respectively. Then, $D$ is the normal connection of the normal bundle $N M$ of $M$. We denote by $\bar{\nabla}$ the connection on the bundle $\left(\otimes^{2} T M\right) \otimes N M$. Then, the covariant derivative of $\sigma$ is defined by

$$
\left(\bar{\nabla}_{X} \sigma\right)(Y, Z)=D_{X}(\sigma(Y, Z))-\sigma\left(\nabla_{X} Y, Z\right)-\sigma\left(Y, \nabla_{X} Z\right) .
$$

The second fundamental form is parallel if $\bar{\nabla} \sigma=0$. The immersion $\varphi$ is said to be $(\lambda-)$ isotropic if $|\sigma(X, X)|$ is equal to a constant $\lambda$ for any unit tangent vector $X$ at each point, and $\varphi$ is said to be $(\lambda-)$ constant isotropic if the function $\lambda$ is constant on $M$. The polarization argument means that $\varphi$ is $\lambda$-isotropic if and only if

$$
\begin{gathered}
\langle\sigma(X, Y), \sigma(Z, W)\rangle+\langle\sigma(X, Z), \sigma(Y, W)\rangle+\langle\sigma(X, W), \sigma(Y, Z)\rangle \\
=\lambda^{2}(\langle X, Y\rangle\langle Z, W\rangle+\langle X, Z\rangle\langle Y, W\rangle+\langle X, W\rangle\langle Y, Z\rangle)
\end{gathered}
$$

for any $X, Y, Z, W \in T M$.

Now, suppose that $N=N(\tilde{c})$ is a complex space form of constant holomorphic sectional curvature $\tilde{c}$ with complex structure tensor $\tilde{J}$. Then, the curvature tensor $\tilde{R}$ of $N(\tilde{c})$ is given by

$$
\begin{aligned}
& \langle\tilde{R}(\tilde{X}, \tilde{Y}) \tilde{Z}, \widetilde{W}\rangle \\
& =\frac{\tilde{c}}{4}\{\langle\tilde{Y}, \tilde{Z}\rangle\langle\tilde{X}, \widetilde{W}\rangle-\langle\tilde{X}, \tilde{Z}\rangle\langle\tilde{Y}, \tilde{W}\rangle+\langle\tilde{J} \tilde{Y}, \tilde{Z}\rangle\langle\tilde{J} \tilde{X}, \widetilde{W}\rangle \\
& \quad-\langle\tilde{J} \tilde{X}, \tilde{Z}\rangle\langle\tilde{J} \tilde{Y}, \widetilde{W}\rangle+2\langle\tilde{X}, \tilde{J} \tilde{Y}\rangle\langle\tilde{J} \tilde{Z}, \widetilde{W}\rangle\},
\end{aligned}
$$

where $\tilde{X}, \tilde{Y}, \tilde{Z}, \widetilde{W} \in T N$. Then, the Gauss and Codazzi equations are respectively given by

$$
\begin{aligned}
& \langle R(X, Y) Z, W\rangle \\
& =\frac{\tilde{c}}{4}\{\langle Y, Z\rangle\langle X, W\rangle-\langle X, Z\rangle\langle Y, W\rangle+\langle\tilde{J} Y, Z\rangle\langle\tilde{J} X, W\rangle \\
& \quad-\langle\tilde{J} X, Z\rangle\langle\tilde{J} Y, W\rangle+2\langle X, \tilde{J} Y\rangle\langle\tilde{J} Z, W\rangle\} \\
& \quad+\langle\sigma(Y, Z), \sigma(X, W)\rangle-\langle\sigma(X, Z), \sigma(Y, W)\rangle, \\
& \quad\left(\bar{\nabla}_{X} \sigma\right)(Y, Z)-\left(\bar{\nabla}_{Y} \sigma\right)(X, Z) \\
& \quad=\frac{\tilde{c}}{4}\{\langle\tilde{J} Y, Z\rangle \tilde{J} X-\langle\tilde{J} X, Z\rangle \tilde{J} Y+2\langle X, \tilde{J} Y\rangle \tilde{J} Z\}^{\perp},
\end{aligned}
$$

where $R$ is the curvature tensor of $M$ and $\{*\}^{\perp}$ denotes the normal component of $\{*\}$. Finally, we mention the following result which is necessary in Sec- 
tion 3.

Proposition 0.1 ([MS]). Let $M$ be a submanifold in a complex space form $N(\tilde{c})$ of constant holomorphic sectional curvature $\tilde{c}$ with complex structure tensor $\tilde{J}$. Then, the following two conditions are equivalent:

(i) $M$ is a circular geodesic submanifold of $N(\tilde{c})$,

(ii) $M$ is nonzero constant isotropic and the second fundamental form $\sigma$ of the immersion satisfies

$$
\left(\bar{\nabla}_{X} \sigma\right)(Y, Z)=\frac{\tilde{c}}{4}\{\langle X, \tilde{J} Y\rangle \tilde{J} Z+\langle X, \tilde{J} Z\rangle \tilde{J} Y\}^{\perp} \quad \text { for any } X, Y, Z \in T M .
$$

\section{$\S 1$. Sufficient conditions for the immersion to be slant.}

Let $\varphi:(M, g) \rightarrow(N,\langle\rangle,, \tilde{J})$ be an isometric immersion. We denote by $\theta_{X}(p)$ the angle between $\tilde{J} \varphi_{*}(X)$ and $\varphi_{*}\left(T_{p} M\right)$ for $X \in T_{p} M$. Then, at any point of $M$, we have

$$
\cos ^{2} \theta_{X}=\sum_{\imath=1}^{n}\left\langle\tilde{J} \varphi_{*}(X), \varphi_{*}\left(\boldsymbol{e}_{\imath}\right)\right\rangle^{2} \quad \text { for } X \in T M \text { with }|X|=1,
$$

where $\left\{\boldsymbol{e}_{i}\right\}$ a local field of orthonormal frames on $M$ and $n=\operatorname{dim} M$. In case $M$ is a Kähler manifold, we choose a local field of unitary frames $\left\{\boldsymbol{u}_{i}\right\}$ in such a way that

$$
\boldsymbol{e}_{\imath}=\frac{1}{\sqrt{2}}\left(\boldsymbol{u}_{i}+\overline{\boldsymbol{u}}_{\imath}\right), \quad J_{M} \boldsymbol{e}_{\imath}=\frac{\sqrt{-1}}{\sqrt{2}}\left(\boldsymbol{u}_{i}-\overline{\boldsymbol{u}}_{\imath}\right) \quad(i=1, \cdots, m),
$$

where $J_{M}$ is a complex structure tensor of $M$ and $m=\operatorname{dim}_{C} M$, and we set

$$
\varphi_{i}=\varphi_{*}\left(\boldsymbol{u}_{\imath}\right), \quad \varphi_{i}=\varphi_{*}\left(\overline{\boldsymbol{u}}_{\imath}\right) \text { for } i=1, \cdots, m .
$$

Since $\cos \theta$ is a continuous function on the unit tangent bundle of $M$, we may assume that $\cos \theta \geqq 0$ in case $\varphi$ is slant.

LEMMA 1.1. (I) $\varphi$ is slant with slant angle $\cos ^{-1} c$ if and only if

$$
\sum_{\imath=1}^{n}\left\langle\tilde{J} \varphi_{*}\left(\boldsymbol{e}_{j}\right), \varphi_{*}\left(\boldsymbol{e}_{\imath}\right)\right\rangle\left\langle\tilde{J} \varphi_{*}\left(\boldsymbol{e}_{k}\right), \varphi_{*}\left(\boldsymbol{e}_{\imath}\right)\right\rangle=c^{2} \boldsymbol{\delta}_{j k}, \quad j, k=1, \cdots, n .
$$

where $c$ is a nonnegative constant.

(II) Suppose that $M$ is an m-dimensional Kähler manifold. If $\varphi^{*} \omega_{N}$ is of type $(1,1)$, where $\omega_{N}$ is the Kähler form of $N$, then, $\varphi$ is slant with slant angle $\cos ^{-1} c$ if and only if

$$
\sum_{i=1}^{m}\left\langle\tilde{J} \varphi_{j}, \varphi_{i}\right\rangle\left\langle\tilde{J} \varphi_{k}, \varphi_{i}\right\rangle=c^{2} \delta_{j k} \quad \text { for } j, k=1, \cdots, m,
$$

where $c$ is a nonnegative constant. 
Proof. (I) Suppose that $\varphi$ is slant with slant angle $\cos ^{-1} c$. Set $X=e_{\mathrm{J}}$ in (1.1). Then we have

$$
\sum_{i=1}^{m}\left\langle\tilde{J} \varphi_{*}\left(\boldsymbol{e}_{j}\right), \varphi_{*}\left(\boldsymbol{e}_{\imath}\right)\right\rangle^{2}=\cos ^{2} \theta_{e_{j}}=c^{2} .
$$

Next, set $X=(1 / \sqrt{2})\left(\boldsymbol{e}_{j}+\boldsymbol{e}_{k}\right)$ for $j \neq k$ in (1.1). Then we have

$$
c^{2}=\cos ^{2} \theta_{X}=\frac{1}{2} c^{2}+\frac{1}{2} c^{2}+\sum_{\imath=1}^{m}\left\langle\tilde{J} \varphi_{*}\left(\boldsymbol{e}_{j}\right), \varphi_{*}\left(\boldsymbol{e}_{\imath}\right)\right\rangle\left\langle\tilde{J} \varphi_{*}\left(\boldsymbol{e}_{k}\right), \varphi_{*}\left(\boldsymbol{e}_{\imath}\right)\right\rangle,
$$

so that we have the equation (1.2). Conversely, if the equation (1.2) holds, then, since $\cos \theta$ is a continuous function on the unit tangent bundle of $M$, we can easily see that $\varphi$ is slant with slant angle $\cos ^{-1} c$.

(II) If $\varphi^{*} \omega_{N}$ is of type $(1,1)$, then $\left\langle\tilde{J} \varphi_{i}, \varphi_{j}\right\rangle=\left\langle\tilde{J} \varphi_{i}, \varphi_{j}\right\rangle=0$ for $\imath, j=1, \cdots, m$. Hence, the equation (1.2) is equivalent to the conditions that, for $j, k=1, \cdots, m$

$$
\operatorname{Re}\left(\sum_{i=1}^{m}\left\langle\tilde{J} \varphi_{j}, \varphi_{i}\right\rangle\left\langle\tilde{J} \varphi_{k}, \varphi_{i}\right\rangle\right)=c^{2} \delta_{j k} \quad \text { and } \quad \operatorname{Im}\left(\sum_{i=1}^{m}\left\langle\tilde{J} \varphi_{j}, \varphi_{i}\right\rangle\left\langle\tilde{J} \varphi_{k}, \varphi_{i}\right\rangle\right)=0,
$$

which are equivalent to the equation (1.3).

q. e. d.

Now, we prove

THEOREM 1.2. Let $\varphi: M \rightarrow N$ be an isometric immersion of an m-dimensional compact Kähler manifold with Kähler form $\omega_{M}$ into a Kähler manifold with Kähler form $\omega_{N}$. Assume that $b_{2}(M)=1$ and $\varphi^{*} \omega_{N}$ is of type $(1,1)$. Then, the following three conditions are equivalent.

(i ) $\operatorname{tr}_{g} \varphi^{*} \omega_{N}=\sqrt{-1} c=$ constant, where $g$ is the Kähler metric on $M$,

(ii) $\varphi^{*} \omega_{N}=(c / m) \omega_{M}$,

(iii) $\varphi$ is slant with slant angle $\cos ^{-1}(|c| / m)$,

Proof. (i) $\Rightarrow$ (ii). If $\operatorname{tr}_{g} \varphi^{*} \omega_{N}=\sqrt{-1} c=$ constant, then $\sum_{l=1}^{m}\left\langle\tilde{J} \varphi_{i}, \varphi_{i}\right\rangle=\sqrt{-1} c$. Since $\varphi^{*} \omega_{N}$ is a closed real $(1,1)$-form and $b_{2}(M)=1$, we have $\left[\varphi^{*} \omega_{N}\right]=a\left[\omega_{M}\right]$ for some real constant $a$, where $\omega_{M}$ is the Kähler form of $M$. Hence, we see that

$$
\left\langle\tilde{J} \varphi_{i}, \varphi_{j}\right\rangle=a \sqrt{-1} \delta_{i j}+\sqrt{-1} \partial_{i} \bar{\partial}_{\jmath} f,
$$

where $f$ is a real valued function on $M$. Taking the trace of this equation, we see that $\Delta f$ is constant, hence $f$ is constant. Therefore, we obtain

$$
\left\langle\tilde{J} \varphi_{i}, \varphi_{j}\right\rangle=\frac{c}{m} \sqrt{-1} \delta_{i j}, \quad \text { for } \imath, j=1, \cdots, m .
$$

which, implies (ii).

(ii) $\Rightarrow$ (iii). This follows from Lemma 1.1 .

(iii) $\Rightarrow$ (i). By Lemma 1.1, we have

$$
\sum_{j=1}^{m} A_{i j} A_{j \bar{k}}=\frac{c^{2}}{m^{2}} \delta_{i k}, \quad \text { for } i, k=1, \cdots, m,
$$


where $A_{\imath j}=\sqrt{-1}\left\langle\tilde{J} \varphi_{i}, \varphi_{j}\right\rangle$, which is a Hermitian matrix. We choose a unitary basis $\left\{\boldsymbol{u}_{j}\right\}$ so that $A_{i j}=\lambda_{i} \delta_{i j}$ with $\lambda_{i} \in \boldsymbol{R}$ for $i=1, \cdots, m$. Then, (1.4) reads as $\lambda_{i}^{2}=c^{2} / m^{2}$, hence $\lambda_{i}= \pm(c / m)$ for $i=1, \cdots, m$. Thus, $\sqrt{-1} \operatorname{tr}_{g} \varphi^{*} \omega_{N}=\operatorname{tr}_{g} A$ is some constant, which is denoted by $\tilde{c}$. By the implications of (i) $\Rightarrow$ (ii) and (ii) $\Rightarrow$ (iii), we have $A_{i j}=(\tilde{c} / m) \delta_{i \jmath}$, which, together with (1.4), yields $|c|=|\tilde{c}|$, hence changing the sign of $c$ if necessary, we have $\operatorname{tr}_{g} \varphi^{*} \omega_{N}=\sqrt{-1} c$, which is constant

q. e. d.

Remark. (1) In case $\operatorname{dim}_{C} M=1, \varphi$ is slant if and only if $\operatorname{tr}_{g} \varphi^{*} \omega_{N}=$ constant, hence we may regard Theorem 1.2 as a natural extension to higher dimensional case because the assumptions that $b_{2}(M)=1$ and $\varphi^{*} \omega_{N}$ is of type $(1,1)$ are automatically satisfied for the case $\operatorname{dim}_{C} M=1$.

(2) Let $M$ be a compact slant submanifold of a Kähler manifold. If $M$ is not a totally real submanifold, then we see that $M$ is even dimensional and $\mathrm{H}^{2 \imath}(M ; \boldsymbol{R}) \neq 0$ for $i=1, \cdots, m$, where $2 m=\operatorname{dim} M$ (see [C]).

An application of Theorem 1.2 will be given in Section 2 .

Next, we give an integral inequality of which the equality occurs only when an immersion is slant under the assumption that $b_{2}(M)=1$.

Assume that $M$ is an $m$-dimensional compact Kähler manifold. Let $U_{p} M=$ $\left\{X \in T_{p} M ;|X|=1\right\}=S^{2 m-1}(1)$ be a unit sphere in $T_{p} M$ and let $U M=\cup_{p \in M} U_{p} M$ be the unit sphere bundle over $M$. By (1.1), we obtain

$$
\int_{X \in U M} \cos ^{2} \theta_{X} d \mu=\frac{\operatorname{vol}\left(S^{2 m-1}(1)\right)}{2 m} \int_{M} \sum_{\imath, j=1}^{2 m}\left\langle\tilde{J} \varphi_{*}\left(\boldsymbol{e}_{j}\right), \varphi_{*}\left(\boldsymbol{e}_{\imath}\right)\right\rangle^{2} * 1,
$$

where $d \mu$ is the volume form of $U M$. The integrand of the right hand side of (1.5) may be rewritten as follows

$$
\begin{aligned}
& \sum_{i, j=1}^{2 m}\left\langle\tilde{J} \varphi_{*}\left(\boldsymbol{e}_{j}\right), \varphi_{*}\left(\boldsymbol{e}_{\imath}\right)\right\rangle^{2} \\
& =\sum_{i, j=1}^{m}\left\{\left\langle\tilde{J} \varphi_{j}, \varphi_{i}\right\rangle\left\langle\tilde{J} \varphi_{j}, \varphi_{i}\right\rangle+\left\langle\tilde{J} \varphi_{j}, \varphi_{i}\right\rangle\left\langle\tilde{J} \varphi_{j}, \varphi_{i}\right\rangle\right\} .
\end{aligned}
$$

On one hand, we have

$$
\varphi^{*} \omega_{N}=\frac{1}{2} \sum_{\imath, j=1}^{m}\left\{\left\langle\tilde{J} \varphi_{i}, \varphi_{\jmath}\right\rangle d z^{\imath} \wedge d z^{\jmath}+2\left\langle\tilde{J} \varphi_{i}, \varphi_{j}\right\rangle d z^{\imath} \wedge d \bar{z}^{\jmath}+\left\langle\tilde{J} \varphi_{i}, \varphi_{j}\right\rangle d \bar{z}^{\imath} \wedge d \bar{z}^{j}\right\} .
$$

Then, a direct computation yields

$$
\begin{aligned}
\varphi^{*} \omega_{N} \wedge \varphi^{*} \omega_{N} \wedge \omega_{M}^{m-2}= & \frac{\omega_{M}^{m}}{m(m-1)} \sum_{i, j=1}^{m}\left\{\left\langle\tilde{J} \varphi_{i}, \varphi_{j}\right\rangle\left\langle\tilde{J} \varphi_{i}, \varphi_{j}\right\rangle\right. \\
& \left.-\left\langle\tilde{J} \varphi_{i}, \varphi_{j}\right\rangle\left\langle\tilde{J} \varphi_{i}, \varphi_{j}\right\rangle-\left\langle\tilde{J} \varphi_{i}, \varphi_{i}\right\rangle\left\langle\tilde{J} \varphi_{\jmath}, \varphi_{j}\right\rangle\right\},
\end{aligned}
$$

which, together with (1.6), implies that 


$$
\begin{aligned}
& \frac{\boldsymbol{\omega}_{M}^{m}}{m(m-1)} \sum_{\imath, j=1}^{2 m}\left\langle\tilde{J} \varphi_{*}\left(\boldsymbol{e}_{j}\right), \varphi_{*}\left(\boldsymbol{e}_{\imath}\right)\right\rangle^{2} \\
& =-\varphi^{*} \omega_{N} \wedge \varphi^{*} \omega_{N} \wedge \omega_{M}^{m-2} \\
& \quad+\frac{2 \omega_{M}^{m}}{m(m-1)} \sum_{\imath, j=1}^{m}\left|\left\langle\tilde{J} \varphi_{\imath}, \varphi_{\jmath}\right\rangle\right|^{2}-\frac{\omega_{M}^{m}}{m(m-1)}\left\{\sum_{i=1}^{m}\left\langle\tilde{J} \varphi_{i}, \varphi_{i}\right\rangle\right\}^{2},
\end{aligned}
$$

where the last term of the right hand side of (1.7) is evaluated from the below as follows

$$
\begin{aligned}
-\int_{M}\left\{\sum_{\imath, \jmath=1}^{m}\left\langle\tilde{J} \varphi_{\imath}, \varphi_{i}\right\rangle\right\}^{2} * 1 & =\int_{M}\left\{\sum_{\imath=1}^{m}\left(\varphi^{*} \omega_{N}\right)\left(\boldsymbol{u}_{\imath}, \overline{\boldsymbol{u}}_{\imath}\right)\right\}^{2} * 1 \\
& \geqq \frac{1}{\operatorname{vol}(M)}\left\{\int_{M} \sum_{\imath=1}^{m}\left(\varphi^{*} \boldsymbol{\omega}_{N}\right)\left(\boldsymbol{u}_{\imath}, \overline{\boldsymbol{u}}_{\boldsymbol{\imath}}\right) * 1\right\}^{2} \\
& =\frac{1}{\operatorname{vol}(M)}\left\{\frac{1}{(m-1) !} \int_{M} \varphi^{*} \omega_{N} \wedge \boldsymbol{\omega}_{M}^{m-1}\right\}^{2},
\end{aligned}
$$

which, together with (1.5), (1.7) and the nonnegativity of the second term of the right hand side of (1.7), implies that

$$
\begin{aligned}
-\frac{2 m}{\operatorname{vol}\left(S^{2 m-1}(1)\right)} \int_{X \in U M} \cos ^{2} \theta_{X} d \mu & \geqq \frac{1}{\operatorname{vol}(M)}\left\{\frac{1}{(m-1) !} \int_{M} \varphi^{*} \omega_{N} \wedge \omega_{M}^{m-1}\right\}^{2} \\
& -\frac{1}{(m-2) !} \int_{M} \varphi^{*} \omega_{N} \wedge \varphi^{*} \omega_{N} \wedge \omega_{M}^{m-2}
\end{aligned}
$$

The equality in (1.8) holds if and only if $\varphi^{*} \omega_{N}$ is of type $(1,1)$ and $\operatorname{tr}_{g} \varphi^{*} \omega_{N}=$ constant. Thus, we have

Proposition 1.3. Let $\varphi:(M, g) \rightarrow N$ be an isometric immersion of a compact Kähler manifold with Kähler form $\omega_{M}$ into a Kähler manifold $N$ with Kähler form $\omega_{N}$. Then, we have

$$
\begin{aligned}
\frac{2 m}{\operatorname{vol}\left(S^{2 m-1}(1)\right)} \int_{X \in U M} \cos ^{2} \theta_{X} d \mu \geqq & \frac{1}{\operatorname{vol}(M)}\left\{\frac{1}{(m-1) !} \int_{M} \varphi^{*} \omega_{N} \wedge \omega_{M}^{m-1}\right\}^{2} \\
& -\frac{1}{(m-2) !} \int_{M} \varphi^{*} \omega_{N} \wedge \varphi^{*} \omega_{N} \wedge \omega_{M}^{m-2},
\end{aligned}
$$

where the equality holds if and only if $\varphi^{*} \omega_{N}$ is of type $(1,1)$ and $\operatorname{tr}_{g} \varphi^{*} \omega_{N}=$ constant. Moreover, if $b_{2}(M)=1$, then the equality holds only if $\varphi$ is slant.

Remark. In the homotopy class of $\varphi$ preserving the volume of $M$, the right hand side of the inequality (1.8) is an invariant quantity. This observation is made more clear by Theorem 2.1 in the next section. 


\section{$\S 2$. Examples of slant immersions into $C P^{N}$.}

In this section, we give the examples of proper slant immersions of a Kähler C-space (that is, a compact simply-connected homogeneous Kähler manifold) $M$ with $b_{2}(M)=1$ into a complex projective space $C P^{N}(\tilde{c})$ of constant holomorphic sectional curvature $\tilde{c}$.

Let $\varphi: M=G / K \rightarrow C P^{N}(\tilde{c})$ be an isometric immersion. The immersion $\varphi$ is called $G$-equivariant if there is a continuous homomorphism $\rho: G \rightarrow S U(N+1)$ such that

$$
\varphi(g \cdot x)=\rho(g) \varphi(x) \quad \text { for any } x \in M, g \in G .
$$

Definition. Let $\varphi: M \rightarrow C P^{N}(\tilde{c})$ be a map from a Kähler manifold $M$ with $\mathrm{H}_{2}(M ; \boldsymbol{Z}) \cong \boldsymbol{Z}$. Denote by $\boldsymbol{\omega}_{M}$ and $\tilde{\boldsymbol{\omega}}$ be the Kähler forms of $M$ and $\boldsymbol{C} P^{N}(\tilde{c})$, respectively. Let $S$ be a positive generator of $\mathrm{H}_{2}(M ; \boldsymbol{Z})$. Then, the degree of $\varphi$ is defined by

$$
\operatorname{deg}(\varphi)=\frac{\tilde{c}}{4 \pi}\left[\varphi^{*} \tilde{\omega}\right](S),
$$

where $\left[\varphi^{*} \widetilde{\omega}\right](S)$ is the evaluation of the cohomology class $\left[\varphi^{*} \widetilde{\omega}\right]$ represented by $\varphi^{*} \widetilde{\omega}$ at $S$.

We prove

THEOREM 2.1. Let $\varphi: M=G / K \rightarrow C P^{N}(\tilde{c})$ be a G-equivariant isometric immersion of an m-dimensional Kähler $C$-space into a complex projective space with Kähler form $\widetilde{\omega}$. Then, $\varphi^{*} \widetilde{\omega}$ is of type $(1,1)$ and $\operatorname{tr}_{g} \tilde{\omega}=$ constant, where $g$ is the Kähler metric on $M$. Moreover, if $b_{2}(M)=1$, then $\varphi$ is slant with slant angle given by

$$
\cos ^{-1}\left(|\operatorname{deg}(\varphi)| \cdot \frac{4 \pi}{\tilde{c} \operatorname{vol}(S)}\right)
$$

where $S$ is a rational curve of $M$ which represents the generator of $\mathrm{H}_{2}(M ; \boldsymbol{Z})$.

Proof. Since $\varphi^{*} \widetilde{\boldsymbol{\omega}}$ is invariant under the action of $G$, we see that $\operatorname{tr}_{g} \varphi^{*} \widetilde{\boldsymbol{\omega}}$ $=$ constant. We denote by $L$ and $\nabla$ the Lie derivation and the covariant differentiation on the tensor bundles of $M$, respectively. Let $U, V, W$ be holomorphic Killing vector fields on $M$. Then, since the $(2,0)$-part $\left(\varphi^{*} \widetilde{\omega}\right)^{2,0}$ of $\varphi^{*} \widetilde{\omega}$ is invariant under the action of $G$, we have

$$
\begin{aligned}
0 & =\left(L_{\bar{U}}\left(\varphi^{*} \widetilde{\omega}\right)^{2,0}\right)(V, W) \\
& =\bar{U} \cdot\left(\varphi^{*} \tilde{\omega}\right)^{2,0}(V, W) \\
& =\left(\nabla_{\bar{U}}\left(\varphi^{*} \tilde{\omega}\right)^{2,0}\right)(V, W) .
\end{aligned}
$$

Therefore, $\left(\varphi^{*} \widetilde{\boldsymbol{\omega}}\right)^{2,0}$ is a holomorphic section of $\otimes^{2} T^{*} M^{1,0}$, where $T^{*} M^{1,0}$ is the holomorphic cotangent bundle of $M$. Since it is known that the first Chern 
class of a Kähler C-space $M$ is positive, it follows from the vanishing theorem that $\left(\varphi^{*} \widetilde{\omega}\right)^{2,0}=\left(\varphi^{*} \widetilde{\omega}\right)^{0,2} \equiv 0$. Moreover, if $b_{2}(M)=1$, it follows from Theorem 1.2 that $\varphi$ is slant. Suppose that $\varphi^{*} \widetilde{\omega}=c \omega_{M}$. Then $\cos \theta=|c|$. On the other hand, we have

$$
\begin{aligned}
\frac{4 \pi}{\tilde{c}} \cdot \operatorname{deg}(\varphi) & =\int_{S} \varphi^{*} \tilde{\omega} \\
& =\int_{S} c \omega_{M} \\
& =c \operatorname{vol}(S),
\end{aligned}
$$

which yields (2.1). q.e.d.

Now, we give the examples of proper slant immersions into $C P^{N}$.

Example 1. Let $\phi_{n, l}^{m}: C P^{m}(\tilde{c}(n, l)) \rightarrow C P^{N(n, l)}(\tilde{c})$ be an $\mathrm{SU}(m+1)$-equivariant full minimal isometric immersion constructed in [O2], where

$$
\tilde{c}(n, l)=\frac{m \tilde{c}}{2 l(n-l)+m n} \text { and }\{(n, l) \in \boldsymbol{Z} \times \boldsymbol{Z} ; n \geqq l \geqq 0\} .
$$

Note that $\psi_{n, l}^{m}$ is holomorphic if and only if $l=0, \phi_{n, l}^{m}$ is anti-holomorphic if and only if $l=n$, and $\phi_{n, l}^{m}$ is totally real if and only if $n=2 l$. By Theorem 2.1, $\phi_{n, l}^{m}$ is slant with slant angle $\cos ^{-1}\left(\left|\operatorname{deg}\left(\phi_{n, l}^{m}\right)\right| \cdot m /(2 l(n-l)+m n)\right)$.

Example 2. Let $f_{p, q}^{m}: \boldsymbol{C} P^{m}(\tilde{c} /(p+q)) \rightarrow \boldsymbol{C} P^{N}(\tilde{c})$ be an $\mathrm{SU}(m+1)$-equivariant isometric immersion defined by

$$
\begin{aligned}
& \left(z_{0}, \cdots, z_{m}\right) \longrightarrow \\
& \left(z_{0}^{p} \bar{z}_{0}^{q}, \cdots, \sqrt{\frac{p !}{\alpha_{0} ! \cdots \alpha_{m} !}} \sqrt{\frac{q !}{\beta_{0} ! \cdots \beta_{m} !}} z_{0}^{\alpha_{0}} \cdots z_{m}^{\alpha_{m}} \bar{z}_{0}^{\beta_{0}} \cdots \bar{z}_{m}^{\beta_{m}}, \cdots, z_{m}^{p} \bar{z}_{m}^{q}\right),
\end{aligned}
$$

where $N=\left(\begin{array}{c}m+p \\ p\end{array}\right)\left(\begin{array}{c}m+q \\ q\end{array}\right)-1, \quad \sum_{\imath=0}^{m} \alpha_{\imath}=p, \quad \sum_{\imath=0}^{m} \beta_{\imath}=q$ and $\alpha_{\imath}, \quad \beta_{i}(0 \leqq \imath \leqq m)$ are nonnegative integers. Let $g: \boldsymbol{C} P^{m}(\tilde{c} /(p+q)) \rightarrow N=\boldsymbol{C} P^{m}(\tilde{c} / p) \times \boldsymbol{C} P^{m}(\tilde{c} / q)$ be a diagonal isometric embedding defined by

$$
g\left(z_{0}, \cdots, z_{m}\right)=\left(z_{0}, \cdots, z_{m} ; \bar{z}_{0}, \cdots, \bar{z}_{m}\right) .
$$

Then, $f_{p, q}^{m}$ is the composition of the Segre embedding, (the $p$-th for the first factor $\left(z_{0}, \cdots, z_{m}\right)$ and the $q$-th for the second factor $\left.\left(\bar{z}_{0}, \cdots, \bar{z}_{m}\right)\right)$ Veronese embeddings and $g$ (for details, see (3.1) in [MO]). Then, since $\operatorname{deg}\left(f_{p, q}^{m}\right)=p-q$, it follows from Theorem 2.1 that $f_{p, q}^{m}$ is slant with slant angle $\cos ^{-1}(|p-q| /(p+q))$.

Before stating the third example of proper slant immersions, we recall the osculating space method of constructing pluriharmonic maps from complex 
manifolds into complex Grassmann manifolds ([OU]). Denote by $G_{k}\left(\boldsymbol{C}^{n}\right)$ a complex Grassmann manifold of $k$-dimensional complex linear subspaces in $\boldsymbol{C}^{n}$. A map $\varphi: M \rightarrow G_{k}\left(C^{n}\right)$ is identified with the bundle $\varphi$ over $M$ of which the fibre $\varphi_{x}$ at $x \in M$ is given by $\varphi(x)$, which is nothing but the pull-back of the universal bundle over $G_{k}\left(\boldsymbol{C}^{n}\right)$ by $\varphi$. Let $\underline{\boldsymbol{C}}^{n+1}=M \times \boldsymbol{C}^{n+1}$ be the trivial bundle over $M$ with standard Hermitian holomorphic vector bundle structure. Then, $\varphi$ is a complex subbundle of $\underline{C}^{n+1}$. We may define the second fundamental form $A^{\varphi}$ of $\varphi$ in $\underline{C}^{n+1}$ and denote by $A_{(1,0)}^{\varphi}$ its $(1,0)$-part. Let $\operatorname{Im} A_{(1,0)}^{\varphi}$ be the image of a bundle homomorphism $A_{(1,0)}^{\varphi}: T M^{1,0} \otimes \varphi \rightarrow \underline{\varphi}^{\perp}$, where $\underline{\varphi}^{\perp}$ is the Hermitian orthogonal complement of $\underline{\varphi}$ in $\underline{\boldsymbol{C}}^{n+1}$. Suppose that $\varphi^{-}$is holomorphic. Set $\varphi_{0}=\varphi$ and define a sequence $\left\{\varphi_{i}\right\}$, inductively, by

$$
\underline{\varphi}_{i}=\operatorname{Im} A_{(1,0)}^{\varphi_{\imath-1}} \quad \text { for } \imath=1,2, \cdots .
$$

By (7.39) in [OU], each $\varphi_{i}$ defines a pluriharmonic map from $M \backslash S_{\varphi_{i}}$ into $G_{k(\imath)}\left(C^{n}\right)$, where $S_{\varphi_{i}}$ is the singularity set of $\varphi_{i}$ and $k(i)$ is a positive integer which depends on $\varphi_{i}$. Moreover, there is a positive integer $r$ such that $\varphi_{r}$ is an anti-holomorphic map and each $\varphi_{i}$ ior $i=1, \cdots, r-1$ is neither holomorphic nor anti-holomorphic. In case $\varphi_{i}$ is an immersion, the pluriharmonic map equation of $\varphi_{i}$ is just the same as

$$
\sigma(Z, \bar{W})=0 \quad \text { for any } Z, W \in T M^{1,0} .
$$

Example 3. Let $\varphi: M=G / K \rightarrow C P^{n-1}(\tilde{c})$ be a G-equivariant Kähler immersion of a Kähler C-space $M$ with $b_{2}(M)=1$. It is known by [NT] that there are many examples of such immersions. Set $\varphi_{0}=\varphi$. Define a sequence $\left\{\varphi_{i}\right\}$ of pluriharmonic maps as above for $i=0,1, \cdots, r$. Then, by the G-equivariance of $\varphi$, we see that each $\varphi_{i}$ is also a G-equivariant immersion of $M$ into $G_{k(i)}\left(C^{n}\right)$ and the induced metric by $\varphi_{i}$ also defines a Kähler metric on $M$. Let $f_{\imath}: G_{k(i)}\left(C^{n}\right) \rightarrow C P^{N}$ be a Plücker embedding and let $\phi_{i}=f_{i^{\circ}} \varphi_{i}: M \rightarrow C P^{N}(\tilde{c})$. Then, by Theorem 2.1, each $\phi_{i}$ for $i=1, \cdots, r-1$ is slant with slant angle $\cos ^{-1}\left(\left|\operatorname{deg}\left(\phi_{i}\right)\right| \cdot(4 \pi / \tilde{c} \operatorname{vol}(S))\right)$, where $S$ is a rational curve of $M$ which represents a positive generator of $\mathrm{H}_{2}(M ; \boldsymbol{Z})$.

\section{$\S 3$. Circular geodesic and slant immersion with constant scalar curvature.}

In this section, suppose that $M$ is an $n$-dimensional submanifold in a nonflat complex space form $N(\tilde{c})$ of constant holomorphic sectional curvature $\tilde{c}$ with complex structure tensor $\tilde{J}$. Let $\left\{\boldsymbol{e}_{i}\right\}(i=1, \cdots, n)$ be a local field of orthonormal frames of $M$.

LEMMA 3.1. Let $\varphi: M \rightarrow N(\tilde{c})$ be a circular geodesic slant ımmertion. Assume that the scalar curvature of $M$ is constant. Then, $\varphi$ is totally real, or the following holds. 


$$
\sum_{\jmath=1}^{n}\left\langle\tilde{J} \boldsymbol{e}_{\jmath}, \sigma_{\imath \jmath}\right\rangle=\sum_{\jmath=1}^{n}\left\langle\tilde{J} \boldsymbol{e}_{\imath}, \sigma_{j j}\right\rangle=0 \quad \text { for } i=1, \cdots, n .
$$

where $\sigma_{\imath \jmath}=\sigma\left(\boldsymbol{e}_{\imath}, \boldsymbol{e}_{j}\right)$.

Proof. Let $\tau$ be the scalar curvature of $M$. Then, by the Gauss equation (0.5) we have

$$
\tau=\frac{\tilde{c}}{4}\left\{n(n-1)+3 \sum_{\imath, j=1}^{n}\left\langle\boldsymbol{e}_{\imath}, \tilde{J} \boldsymbol{e}_{\jmath}\right\rangle^{2}\right\}+\sum_{\imath, \jmath=1}^{n}\left\{\left\langle\sigma_{i i}, \sigma_{j j}\right\rangle-\left\langle\sigma_{\imath \jmath}, \sigma_{\imath \jmath}\right\rangle\right\} .
$$

On one hand, since $\varphi$ is constant isotropic, we have

$$
\sum_{i, j=1}^{n}\left\{\left\langle\sigma_{i i}, \sigma_{j j}\right\rangle+2\left\langle\sigma_{\imath \jmath}, \sigma_{\imath \jmath}\right\rangle\right\}=n(n+2) \lambda^{2},
$$

where $\lambda$ is a (non-zero) isotropic constant. It follows from (3.2), (3.3) and the present assumptions that each of the quantities $\Sigma_{\imath, \jmath}\left\langle\sigma_{\imath \jmath}, \sigma_{\imath \jmath}\right\rangle$ and $\Sigma_{\imath, \jmath}\left\langle\sigma_{i i}, \sigma_{j j}\right\rangle$ is constant. Using Proposition 0.1 we compute

$$
\begin{aligned}
& 0=2 \sum_{\imath, \jmath}\left\langle\left(\bar{\nabla}_{\boldsymbol{e}_{k}} \sigma\right)\left(\boldsymbol{e}_{\imath}, \boldsymbol{e}_{j}\right), \sigma_{\imath \jmath}\right\rangle=\tilde{c} \sum_{\imath, \jmath}\left\langle\boldsymbol{e}_{k}, \tilde{J} \boldsymbol{e}_{\imath}\right\rangle\left\langle\tilde{J} \boldsymbol{e}_{\jmath}, \sigma_{\imath \jmath}\right\rangle, \\
& 0=2 \sum_{\imath, \jmath}\left\langle\left(\bar{\nabla}_{\boldsymbol{e}_{k}} \sigma\right)\left(\boldsymbol{e}_{\imath}, \boldsymbol{e}_{\imath}\right), \sigma_{j j}\right\rangle=\tilde{c} \sum_{\imath, \jmath}\left\langle\boldsymbol{e}_{k}, \tilde{J} \boldsymbol{e}_{\imath}\right\rangle\left\langle\tilde{J} \boldsymbol{e}_{\imath}, \sigma_{j j}\right\rangle,
\end{aligned}
$$

where $k=1, \cdots, n$. Since $\varphi$ is slant, we know that

$$
\sum_{\imath}\left\langle\boldsymbol{e}_{\jmath}, \tilde{J} \boldsymbol{e}_{\imath}\right\rangle\left\langle\tilde{J} \boldsymbol{e}_{\imath}, \boldsymbol{e}_{k}\right\rangle=s^{2} \boldsymbol{\delta}_{j k} \quad \text { for } j, k=1, \cdots, n,
$$

where $\cos ^{-1}|s|$ is a constant slant angle. Suppose that $\varphi$ is not totally real, so that $s \neq 0$. Then, it follows from (3.4), (3.5) and (3.6) that the equation (3.1) holds.

q. e. d.

LEMMA 3.2. Let $\varphi: M \rightarrow N(\tilde{c})$ be as in Lemma 3.1. If $\varphi$ is not totally real, then we have

$$
\sum_{k, j}\left\langle\boldsymbol{\sigma}_{k k}, \boldsymbol{\sigma}_{j l}\right\rangle\left\langle\tilde{J} \boldsymbol{e}_{\imath}, \boldsymbol{e}_{\jmath}\right\rangle=\sum_{k}\left\langle\boldsymbol{\sigma}_{k k}, \tilde{J} \boldsymbol{\sigma}_{i l}\right\rangle+\frac{\tilde{c}}{2}\left(1-s^{2}\right)\left\langle\boldsymbol{e}_{l}, \tilde{J} \boldsymbol{e}_{\imath}\right\rangle,
$$

for $i, l=1, \cdots, n$, where $\cos ^{-1}|s|$ is a constant slant angle. In particular, we have

$$
\sum_{k, j}\left\langle\sigma_{k k}, \sigma_{j j}\right\rangle=\frac{\tilde{c}}{2}\left(1-s^{2}\right) n
$$

Proof. By Lemma 3.1, we know that $\Sigma_{k}\left\langle\tilde{J} e_{\imath}, \sigma_{k k}\right\rangle=0$ for $i=1, \cdots, n$. Differentiating this equation covariantly, and using Proposition 0.1 , we compute 


$$
\begin{aligned}
0= & \sum_{k}\left\langle\tilde{J} \sigma_{i l}, \sigma_{k k}\right\rangle+\sum_{k}\left\langle\tilde{J} \boldsymbol{e}_{\imath},\left(\bar{\nabla}_{e_{l}} \sigma\right)\left(\boldsymbol{e}_{k}, \boldsymbol{e}_{k}\right)-\sum_{\jmath}\left\langle\sigma_{k k}, \sigma_{l \jmath}\right\rangle \boldsymbol{e}_{\jmath}\right\rangle \\
= & \sum_{k}\left\langle\tilde{J} \sigma_{i l}, \sigma_{k k}\right\rangle-\sum_{k, \jmath}\left\langle\sigma_{k k}, \sigma_{j l}\right\rangle\left\langle\tilde{J} \boldsymbol{e}_{\imath}, \boldsymbol{e}_{\jmath}\right\rangle \\
& +\frac{\tilde{c}}{2}\left\{\sum_{k}\left\langle\boldsymbol{e}_{l}, \tilde{J} \boldsymbol{e}_{k}\right\rangle\left\langle\tilde{J} \boldsymbol{e}_{k}, \tilde{J} \boldsymbol{e}_{\imath}\right\rangle-\sum_{k, \jmath}\left\langle\boldsymbol{e}_{l}, \tilde{J} \boldsymbol{e}_{k}\right\rangle\left\langle\tilde{J} \boldsymbol{e}_{k}, \boldsymbol{e}_{\jmath}\right\rangle\left\langle\boldsymbol{e}_{\jmath}, \tilde{J} \boldsymbol{e}_{\imath}\right\rangle\right\},
\end{aligned}
$$

which, together with (3.6), yields (3.7). Multiplying the both sides of (3.7) by $\left\langle\tilde{J} \boldsymbol{e}_{\imath}, \boldsymbol{e}_{l}\right\rangle$ and summing up with respect to the indices $i$ and $l$, we obtain (3.8).

q. e. d.

Now, we prove

ThEOREM 3.3. Let $\varphi: M \rightarrow N(\tilde{c})$ be a circular geodesic slant immersion. Assume that the scalar curvature of $M$ is constant. Then, the second fundamental form of $\varphi$ is parallel.

Proof. Suppose that $\varphi$ is not totally real. We compute $\Sigma_{k} \tilde{R}\left(\boldsymbol{e}_{\imath}, \boldsymbol{e}_{j}\right) \sigma_{k k}$ by using Lemma 3.1 and Lemma 3.2.

$$
\begin{aligned}
& \sum_{k} \tilde{\nabla}_{\boldsymbol{e}_{j}} \boldsymbol{\sigma}_{k k} \\
& =\sum_{k}\left\{\left(\bar{\nabla}_{\boldsymbol{e}_{j}} \boldsymbol{\sigma}\right)\left(\boldsymbol{e}_{k}, \boldsymbol{e}_{k}\right)+2 \boldsymbol{\sigma}\left(\nabla_{\boldsymbol{e}_{j}} \boldsymbol{e}_{k}, \boldsymbol{e}_{k}\right)-\sum_{m}\left\langle\boldsymbol{\sigma}_{k k}, \boldsymbol{\sigma}_{\jmath m}\right\rangle \boldsymbol{e}_{m}\right\} \\
& =\frac{\tilde{c}}{2}\left\{\sum_{k}\left\langle\boldsymbol{e}_{\jmath}, \tilde{J} \boldsymbol{e}_{k}\right\rangle \tilde{J} \boldsymbol{e}_{k}-s^{2} \boldsymbol{e}_{j}\right\}+\sum_{k}\left\{2 \sigma\left(\nabla_{\boldsymbol{e}_{j}} \boldsymbol{e}_{k}, \boldsymbol{e}_{k}\right)-\sum_{m}\left\langle\boldsymbol{\sigma}_{k k}, \boldsymbol{\sigma}_{\jmath m}\right\rangle \boldsymbol{e}_{m}\right\} .
\end{aligned}
$$

At a point where $\nabla_{e_{i}} \boldsymbol{e}_{\jmath}=0$ for $i, j=1, \cdots, n$, we have

$$
\begin{aligned}
& \sum_{\boldsymbol{k}} \tilde{\nabla}_{\boldsymbol{e}_{i}} \tilde{\nabla}_{e_{j}} \boldsymbol{\sigma}_{k k} \\
& =\frac{\tilde{c}}{2}\left\{\sum_{k}\left\{\left\langle\boldsymbol{\sigma}_{\imath \jmath}, \tilde{J} \boldsymbol{e}_{k}\right\rangle \tilde{J} \boldsymbol{e}_{k}+\left\langle\boldsymbol{e}_{\jmath}, \tilde{J} \boldsymbol{\sigma}_{i k}\right\rangle \tilde{J} \boldsymbol{e}_{k}+\left\langle\boldsymbol{e}_{\jmath}, \tilde{J} \boldsymbol{e}_{k}\right\rangle \tilde{J} \boldsymbol{\sigma}_{i k}\right\}-s^{2} \boldsymbol{\sigma}_{\imath \jmath}\right\} \\
& +\sum_{k}\left\{2 \boldsymbol{\sigma}\left(\nabla_{\boldsymbol{e}_{i}} \nabla_{\boldsymbol{e}_{j}} \boldsymbol{e}_{k}, \boldsymbol{e}_{k}\right)-\frac{\tilde{c}}{2} \sum_{m}\left\langle\boldsymbol{e}_{\imath}, \tilde{J} \boldsymbol{e}_{k}\right\rangle\left\langle\tilde{J} \boldsymbol{e}_{k}, \boldsymbol{\sigma}_{\jmath m}\right\rangle \boldsymbol{e}_{m}-\Sigma\left\langle\sigma_{k k}, \sigma_{\jmath m}\right\rangle \boldsymbol{\sigma}_{\imath m}\right\},
\end{aligned}
$$

where we have used (3.1). Then, we obtain

$$
\begin{aligned}
& \sum_{k} \tilde{R}\left(\boldsymbol{e}_{\imath}, \boldsymbol{e}_{j}\right) \sigma_{k k} \\
& =\frac{\tilde{c}}{2} \sum_{k}\left\{\left\langle\boldsymbol{e}_{\jmath}, \tilde{J} \boldsymbol{\sigma}_{i k}\right\rangle \tilde{J} \boldsymbol{e}_{k}-\left\langle\boldsymbol{e}_{\imath}, \tilde{J} \sigma_{j k}\right\rangle \tilde{J} \boldsymbol{e}_{k}+\left\langle\boldsymbol{e}_{\jmath}, \tilde{J} \boldsymbol{e}_{k}\right\rangle \tilde{J} \sigma_{i k}-\left\langle\boldsymbol{e}_{\imath}, \tilde{J} \boldsymbol{e}_{k}\right\rangle \tilde{J} \sigma_{j k}\right\} \\
& \quad-\frac{\tilde{c}}{2} \sum_{k, m}\left\{\left\langle\boldsymbol{e}_{\imath}, \tilde{J} \boldsymbol{e}_{k}\right\rangle\left\langle\tilde{J} \boldsymbol{e}_{k}, \sigma_{\jmath m}\right\rangle-\left\langle\boldsymbol{e}_{\jmath}, \tilde{J} \boldsymbol{e}_{k}\right\rangle\left\langle\tilde{J} \boldsymbol{e}_{k}, \sigma_{\imath m}\right\rangle\right\} \boldsymbol{e}_{m} \\
& \quad-\sum_{k, m}\left\{\left\langle\boldsymbol{\sigma}_{k k}, \boldsymbol{\sigma}_{\jmath m}\right\rangle \boldsymbol{\sigma}_{\imath m}-\left\langle\boldsymbol{\sigma}_{k k}, \boldsymbol{\sigma}_{\imath m}\right\rangle \boldsymbol{\sigma}_{\jmath m}\right\},
\end{aligned}
$$


because $\sigma_{\imath \jmath}=\sigma_{j i}$ and

$$
\sum_{k}\left\{\boldsymbol{\sigma}\left(\nabla_{\boldsymbol{e}_{i}} \nabla_{\boldsymbol{e}_{j}} \boldsymbol{e}_{k}, \boldsymbol{e}_{k}\right)-\boldsymbol{\sigma}\left(\nabla_{\boldsymbol{e}_{j}} \nabla_{\boldsymbol{e}_{i}} \boldsymbol{e}_{k}, \boldsymbol{e}_{k}\right)\right\}=\sum_{k, m}\left\langle R\left(\boldsymbol{e}_{\imath}, \boldsymbol{e}_{j}\right) \boldsymbol{e}_{k}, \boldsymbol{e}_{m}\right\rangle \boldsymbol{\sigma}_{m k}=0 .
$$

On the other hand, since $\tilde{R}$ has a particular form (0.4), we have

$$
\sum_{k} \tilde{R}\left(\boldsymbol{e}_{\imath}, \boldsymbol{e}_{j}\right) \sigma_{k k}=\frac{\tilde{c}}{2}\left\langle\boldsymbol{e}_{\imath}, \tilde{J} \boldsymbol{e}_{\jmath}\right\rangle \sum_{k} \tilde{J} \sigma_{k k},
$$

where we have used (3.1). Therefore, multiplying the both of the right hand sides of (3.9) and (3.10) by $\left\langle\boldsymbol{e}_{\imath}, \tilde{J} \boldsymbol{e}_{\jmath}\right\rangle$ and summing up with respect to the indices $i$ and $j$, we obtain

$$
\begin{aligned}
& \frac{\tilde{c}}{2} s^{2} n \sum_{k} \tilde{J} \sigma_{k k} \\
& =\frac{\tilde{c}}{2} \sum_{k}\left\{\sum_{\imath, \jmath}\left\langle\boldsymbol{e}_{\imath}, \tilde{J} \boldsymbol{e}_{\jmath}\right\rangle\left\langle\left\langle\boldsymbol{e}_{\jmath}, \tilde{J} \sigma_{i k}\right\rangle-\left\langle\boldsymbol{e}_{\imath}, \tilde{J} \sigma_{j k}\right\rangle\right\} \tilde{J} \boldsymbol{e}_{k}-2 s^{2} \tilde{J} \boldsymbol{\sigma}_{k k}\right\} \\
& \quad-2 \sum_{\imath, j, k, m}\left\langle\boldsymbol{\sigma}_{k k}, \sigma_{\jmath m}\right\rangle\left\langle\boldsymbol{e}_{\imath}, \tilde{J} \boldsymbol{e}_{\jmath}\right\rangle \boldsymbol{\sigma}_{\imath m},
\end{aligned}
$$

where we have used (3.1). Then, using (3.7) and (3.11), we compute

$$
\begin{aligned}
& \frac{\tilde{c}}{2} s^{2} n \sum_{k, l}\left\langle\sigma_{k k}, \sigma_{l l}\right\rangle \\
& =\frac{\tilde{c}}{2} s^{2} n \sum_{k, l}\left\langle\tilde{J} \sigma_{k k}, \tilde{J} \sigma_{l l}\right\rangle \\
& =-\tilde{c} s^{2} \sum_{k, l}\left\langle\sigma_{k k}, \sigma_{l l}\right\rangle \\
& \quad+2 \sum_{\imath, l, m}\left\{\sum_{k}\left\langle\sigma_{k k}, \tilde{J} \sigma_{\imath m}\right\rangle+\frac{\tilde{c}}{2}\left(1-s^{2}\right)\left\langle\boldsymbol{e}_{m}, \tilde{J} \boldsymbol{e}_{\imath}\right\rangle\right\}\left\langle\sigma_{\imath m}, \tilde{J} \sigma_{l l}\right\rangle \\
& =-\tilde{c} s^{2} \sum_{k, l}\left\langle\sigma_{k k}, \sigma_{l l}\right\rangle+2 \sum_{\imath, k, l, m}\left\langle\sigma_{k k}, \tilde{J} \sigma_{\imath m}\right\rangle\left\langle\sigma_{\imath m}, \tilde{J} \sigma_{l l}\right\rangle,
\end{aligned}
$$

hence

$$
\frac{\tilde{c}}{2} s^{2}(n+2) \sum_{k, l}\left\langle\sigma_{k k}, \sigma_{l l}\right\rangle=-2 \sum_{\imath, m}\left\langle\sum_{k} \sigma_{k k}, \tilde{J} \sigma_{\imath m}\right\rangle^{2} \leqq 0 .
$$

If $\tilde{c}<0$, then, by (3.8) we have $s^{2}=1$, so that $M$ is a Kähler submanifold. If $\tilde{c}>0$, then, by (3.8) and (3.12) we also have $s^{2}=1$, so that $M$ is a Kähler submanifold. Thus, we have proved that $M$ is a totally real or a Kähler submanifold. Then, we see that the second fundamental form of $\varphi$ is parallel. q.e.d.

COROLlaRY 3.4. Let $\varphi: M \rightarrow N(\tilde{c})$ be a circular geodesic slant immersion. Assume that the mean curvature of $\varphi$ is constant. Then, the second fundamental form of $\varphi$ is parallel. 
Proof. Let $H$ be the mean curvature of $\varphi$. Then, $H^{2}=1 / n^{2} \Sigma_{\imath, j}\left\langle\sigma_{i i}, \sigma_{j j}\right\rangle$. It follows from (3.2) and (3.3) that $H$ is constant if and only if $\tau$ is constant. Then, Corollary 3.4 follows from Theorem 3.3.

q. e. d.

THEOREM 3.5. Let $\varphi: M \rightarrow N(\tilde{c})$ be a circular geodesic immersion of a surface. Then, the following four conditons are mutually equivalent:

(1) The mean curvature of $\varphi$ is constant,

(2) The second fundamental form of $\varphi$ is parallel,

(3) The slant angle (i.e., the Kähler angle) of $\varphi$ is constant,

(4) The scalar curvature of $M$ is constant.

Proof. The equivalences among (2), (3) and (4) are already proved in [MU]. We only prove the equivalence between (1) and (3). Since $\varphi$ is constant isotropic (cf. (3.3)), (1) is equivalent to saying that

$$
\sum_{i, \jmath}\left\{\left\langle\sigma_{i i}, \sigma_{j j}\right\rangle-\left\langle\sigma_{\imath \jmath}, \sigma_{\imath \jmath}\right\rangle\right\}=2\left\{\left\langle\sigma_{11}, \sigma_{22}\right\rangle-\left\langle\sigma_{12}, \sigma_{12}\right\rangle\right\}
$$

is constant. However, by Proposition 0.1 we have

$$
\boldsymbol{e}_{\imath}\left\{\left\langle\sigma_{11}, \sigma_{22}\right\rangle-\left\langle\sigma_{12}, \sigma_{12}\right\rangle\right\}=\frac{\tilde{c}}{4} \boldsymbol{e}_{i}\left\langle\boldsymbol{e}_{1}, \tilde{J} \boldsymbol{e}_{2}\right\rangle^{2} \quad \text { for } i=1,2,
$$

hence (1) is equivalent to (3).

q.e.d.

\section{REFERENCES}

[BO] Bando, S. And Ohnita, Y., Minimal 2-spheres with constant curvature in $P_{n}(C)$, J. Math. Soc. Japan, 39 (1987), 477-487.

[BJRW] Bolton, J., Jensen, G.R., Rigoli, M. ANd Woodward, L. M., On conformal minimal immersions of $S^{2}$ into $C P^{n}$, Math. Ann. 279 (1988), 599-620.

[C] ChEN, B. Y., Geometry of slant submanifolds, Katholieke Universiteit Leuven, 1990.

[CT] Chen, B. Y. AND TAZAwA, Y., Slant submanifolds in complex Euclidean space, Tokyo J. Math. 14 (1991), 101-120.

[CW] Chern, S.S. And Wolfson, J.G., Minimal surfaces by moving frames, Amer. J. Math. 105 (1983), 59-83.

[M] MaEdA, S., Differential geometry of constant mean curvature submanifolds, Mem. Fac. Gen. Ed. Kumamoto Univ. Nat. Sci. 24 (1989), 7-39.

[MO] MAEDA, S. AND OHNitA, Y., Helical geodesic immersions into complex space forms, Geom. Dedicata 30 (1989), 93-114.

[MS] MAEDA, S. AND SATO, N., On submanifolds all of whose geodesics are circles in a complex space form, Kodai Math. J. 6 (1983), 157-166.

[MU] MaEda, S. AND Udagawa, S., Surfaces with constant Kähler angle all of whose geodesics are circles in a complex space form, Tokyo J. Math. 13 (1990), 341351 . 
[NT] NAKagawa, H. AND TAKagi, R., On locally symmetric Kähler submanifolds in a complex projective space, J. Math. Soc. Japan, 28 (1976), 638-667.

[O1] OhNita, Y., Minimal surfaces with constant curvature and Kähler angle in complex space forms, Tsukuba J. Math. 13 (1989), 191-207.

[O2] OHNitA, Y., Homogeneous harmonic maps into complex projectve spaces, Tokyo J. Math. 13 (1990), 87-116.

[OU] Ohnita, Y. AND Udagawa, S., Complex-analyticity of pluriharmonic maps and their constructions, Prospects in Complex Geometry, Proceedings, Katata/Kyoto 1989, Lect. Notes in Math. 1468, Springer-Verlag, 371-407.

[S] Sakamoto, K., Planar geodesic immersions, Tôhoku Math. J. 29 (1977), 25-56.

[T] Tazawa, Y., Slant immersions into complex Euclidean spaces with higher dimensions and codimensions, preprint.

Department of Mathematics, Nagoya Institute of Technology GoKISO, NAGOYA 466, JAPAN

Department of Mathematics, Tokyo Metropolitan University HACHI-ÔJI, TOKYO 192-03, JAPAN

Department of Mathematics, School of Medicine, Nihon University ITABASHI, TOKYO 173 , JAPAN 